notation and the balance of the material, the course was written in the early sixties and has not changed its shape since then. The first five chapters, which occupy half of the main text, give an introduction to weak interactions. There is a great deal of historical detail on the Fermi and Gamow-Teller theories. Parity non-conservation is treated at length, as if it were a brand new phenomenon, before a long and circumstantial chapter on the V-A theory. The weak interactions of elementary particles are given a reasonable amount of space, but with an alarming vagueness. This is certainly not the place to read about the Cabibbo theory, for instance. Its results are mentioned, but after a page and a half of introduction the author announces that "we do not have space to introduce the SU3 computation". Surely he could introduce Clebsch-Gordan coefficients, by analogy with SU2, to justify the precise numerical predictions of the theory which he tabulates.

The second half of the main text is devoted to the chief business of the book, a review of beta decay and muon capture as tools for nuclear structure physics. The presentation is pedestrian, but the details of individual experiments are given at unusual length. Although most of the reference lists stop in about 1969 , there is a chapter which deals with more recent developments, such as the investigation of second class currents in the transitions of mirror nuclei.

Because of the detail in the later chapters, and because of the patiently long-winded explanations of many technical points, this book should be very useful to experimental students of nuclear physics. But they should come to it after a simple course on the weak interactions of elementary particles, based for instance on some of the excellent reports published by CERN, Geneva (Cabibbo and Veltman: CERN 65-30; and J. S. Bell: CERN 72-4).

D. J. Miller

\section{Crust of the Earth}

Structural Geomorphology. By J. Tricart. Translated by S. H. Beaver and E. Derbyshire. Pp. xiii +305 . (Geographies for Advanced Study.) (Longman: London, April 1974.) $£ 4.75$.

Professor TRICHART's book, published in France in 1968, has been admirably translated by two geographers from the University of Keele. It is intended not only for students taking a first degree in geography but also for specialists in the "cognate disciplines" of geology, pedology, ecology and planning. The book is built round the premise, stated on the first page of the introduction, that the surface features of the lithosphere have been moulded by the interaction of internal and external forces at work in the Earth's crust and the author recognises a close relationship between characteristic landforms and large-scale crustal structures. The first chapter deals with the distinctions between continents and oceans, the second with geosynclines and fold belts, the third with platform areas and the final chapter with faults and volcanoes. Stress is laid on the fact that landscapes evolve over long periods.

This scheme of treatment, together with Professor Trichart's excellent aphorism (page 22) that "nature herself is a unified whole" raises the hope that the book, by bringing geological and geographical thinking to bear on a subject involving both disciplines, may illuminate the whole field of geomorphology. Where details are concerned, the book does indeed provide many clear and well illustrated examples of the relationship between structure and landform.

Further reading, however, raises serious doubts about the geological treatment. The author's views of crustal evolution have much in common with those of Soviet geologists who discount the possibility that large-scale horizontal displacements of continents have taken place during the past few hundred million years. This view is seldom expressed today in English or American works and a reasoned exposition of it might have been welcome. But it is disconcerting to find no discussion of the evidence concerning seafloor spreading (the mid-Atlantic ridge is referred to only once, on page 36 , as a structure "somewhat similar to an island arc") and even more so to find on page 45 the statement that "despite the precision of the techniques, no measurements yet made have shown the slightest signs of change in the relative positions of Europe and North America, which should have occurred if the two continents are drifting apart".

This statement, made without any qualification, is the more surprising in that classic palaeomagnetic studies by Runcorn, Tarling and others are cited in the bibliography. Errors of fact are not uncommon: for example, the Moho, though defined as the base of the crust on page 41 , is referred to on the next page as a discontinuity within the crust: the schistes lustrés are described as a flysch facies. More important, perhaps, from a student's point of view is the lack of a stratigraphical table or any other data from which he can discover the relative ages of the geological systems mentioned, the absence of any account of landforms associated with depositional processes, and the cursory treatment meted out to rift valleys. Since the geological aspect is fundamental to the author's approach, I feel that defects of this kind undermine the whole structure of the book.

J. WATSON

\section{Excited molecules}

Excited States. Vol. 1 Edited by Edward C. Lim. Pp. xii +347 . (Academic: New York and London, January 1974.) $\$ 24.50 ; £ 11.75$.

INTEREST in the structure of electronically excited species in the gas phase has been growing apace in the last two or three decades. A discussion of the Faraday Society in 1963, on just that subject, was an undoubted success. Somewhat earlier (1955) had come a text by Laidler on The Chemical Kinetics of Excited States, and Reid's book (1957), Excited States in Chemistry and Biology, was a stimulating early work.

The present volume, it might be argued, is not one but six. Certainly, its six chapters differ considerably in subject matter. The first, by G. Wilse Robinson, deals with radiationless transitions, whereas the remaining five deal with topics that involve emission or absorption of radiation. Robinson's chapter is much the most readable of the six. The second chapter, by M. A. El-Sayed, contains a comprehensive review of phosphorescence microwave double resonance spectroscopy (PMDR). It seems from this article that there will be a considerable increase in the number of abbreviations that infest the literature.

The third chapter, by Robin $M$. Hochstrasser and Paras N. Prasad, deals with optical spectra and relaxation in molecular solids. It outlines various aspects of the electronic spectra of solids (molecular crystals and mineral crystals) and contains detailed discussion on phonon interactions that have not previously been treated in detail. The fourth chapter, by Wolfgang Liplay, is an excellent account of the determination of dipole moments and polarisabilities of molecules in excited electronic states. The fifth chapter, by C. J. Selisker, O. S. Khalil and S. P. McGlynn, treats the luminescence of polar aromatic molecules; and the sixth, by A. J. Duben, L. Goodman and M. Koyanagi, deals with interstate interactions in aromatic aldehydes and ketones.

The book is very well produced. I found perhaps half-a-dozen printer's errors, none of them of great import. Further volumes are planned.

$$
\text { A. D. WALSH }
$$

